\title{
Surface Charge Compensation for a Highly Charged Ion Emission Microscope
}

\author{
J. W. McDonald, A. V. Hamza, M. W. Newman, \\ J. P. Holder, and D. H. G. Schneider \\ Lawrence Livermore National Laboratory \\ T. Schenkel \\ E. O. Lawrence Berkeley National Laboratory
}

\begin{abstract}
A surface charge compensation electron flood gun has been added to the Lawrence Livermore National Laboratory (LLNL) highly charged ion (HCI) emission microscope. HCI surface interaction results in a significant charge residue being left on the surface of insulators and semiconductors. This residual charge causes undesirable aberrations in the microscope images and a reduction of the Time-Of-Flight (TOF) mass resolution when studying the surfaces of insulators and semiconductors. The benefits and problems associated with HCI microscopy and recent results of the electron flood gun enhanced HCI microscope are discussed.
\end{abstract}

\section{INTRODUCTION}

In the last few years increasing demands of the semiconductor industry for materials characterization at high spatial resolution have driven the instrument development field. To meet the increasing demands of the semiconductor industry for materials characterization at very high spatial resolution an impressive array of instruments have been developed (transmission electron microscopy, scanning tunneling electron microscopy, etc.). There is an equally impressive array of instruments and techniques available to determine material composition (secondary ion mass spectroscopy, Auger electron spectroscopy, photoelectron spectroscopy, etc.). The LLNL HCI driven emission microscope provides both high spatial resolution and very sensitive compositional analysis simultaneously it has been described in detail by Hamza et al. ${ }^{1}$. Emission microscopes are a special class of electron microscopes that accelerate and image low energy electrons and other charged particles from a planar surface. The history of emission microscopes began more than 100 years ago (see reference ${ }^{2}$ ). The HCI emission microscope described here is a combination of two emission microscopes developed in the 1960s. The first an $\mathrm{Ar}^{+}$ion beam induced electron emission microscope $\mathrm{e}^{3}$ where the kinetic emission of electrons forms an image. The second a secondary ion emission microscope ${ }^{4}$ where an ion beam is used to sputter secondary ions, which are imaged. In the present instrument electrons and secondary ions are imaged and the mass of the secondary ions is determined by time of flight. Thus, a variety of contrast imaging modes are available.

The emission microscope described here uses HCIs as the excitation source. The highly charged ions bring four crucial advantages, a large secondary electron yield, high secondary ion yields, high ionization probability of the secondary emission, and high molecular yields. 
Highly charged ions carry considerable potential energy (the sum of the ionization potentials of the highly charged ion) to surfaces and this energy is released in the first few femtoseconds of surface interaction resulting in a highly localized energy deposition in the first few atomic layers of the surface ${ }^{5}$. In contrast is the case of singly charged ion induced secondary ion mass spectroscopy (SIMS) where most of the energy transferred to the surface comes from the kinetic energy of the projectile ion. This kinetic transfer is a multistep, relatively low cross section process that is distributed across a comparatively deep collision and is not localized to the surface layers. The localized energy deposition from a HCI/surface interaction results in very high (100s of electrons per primary ion) electron ${ }^{6,7}$ and (greater than one) secondary ion yields ${ }^{8}$. The ratio of the secondary ion yield to the secondary neutral yield gives ionization probabilities of $10 \%$ for $\mathrm{HCI}$ excitation ${ }^{9}$. This very high electron yield is employed to produce the very high spatial resolution. Present photoelectron emission microscopes can achieve less than $10 \mathrm{~nm}$ resolution imaging single electron events ${ }^{10}$. The high secondary ion yield, particularly the high secondary molecular ion yield, and the high ionization probability that $\mathrm{HCI}$ excitation produces offer the potential of providing highly sensitive compositional information at high spatial resolution.

Unfortunately, the potential energy associated with the HCI surface interaction also involves charge deposition. This charge is of little consequence when the HCI is interacting with a conductor where the high electron mobility can dissipate the charge. However, in the case of semiconductors and insulators the surface charges up. This surface charging leads to serious distortions in the images produced in the HCI emission microscope as well as a significant reduction in the resolution of the secondary ion mass time-offlight spectra. This paper addresses one way around this charging problem.

\section{EXPERIMENT}

In this microscope a beam of highly charged ions in a single charge state (i.e. $\mathrm{Xe}^{44+}$ ) from the LLNL EBIT $^{11}$ is focused onto the sample to be analyzed. The secondary electrons or ions are accelerated and focused onto a channel plate detector with a resistive anode position sensitive imaging system. The experimental setup is described in detail by Hamza in reference 1. The main disadvantage of employing HCIs as the excitation source is the charge built up on the surface of the sample when studying semiconductors and insulators. In an effort to neutralize the charge left on the surface we have installed an electron flood gun. The electron flood gun, shown schematically in figure 1 (left) was put together from parts on hand in our laboratory. The timing of the electron flood gun emission, sample bias, HCIs, and data acquisition were controlled by a timing sequencer. The filament current, cathode bias and duty cycle between the $\mathrm{HCI}$ and electron exposure were varied to determine the optimum operating conditions for 
each sample and effective ion current.
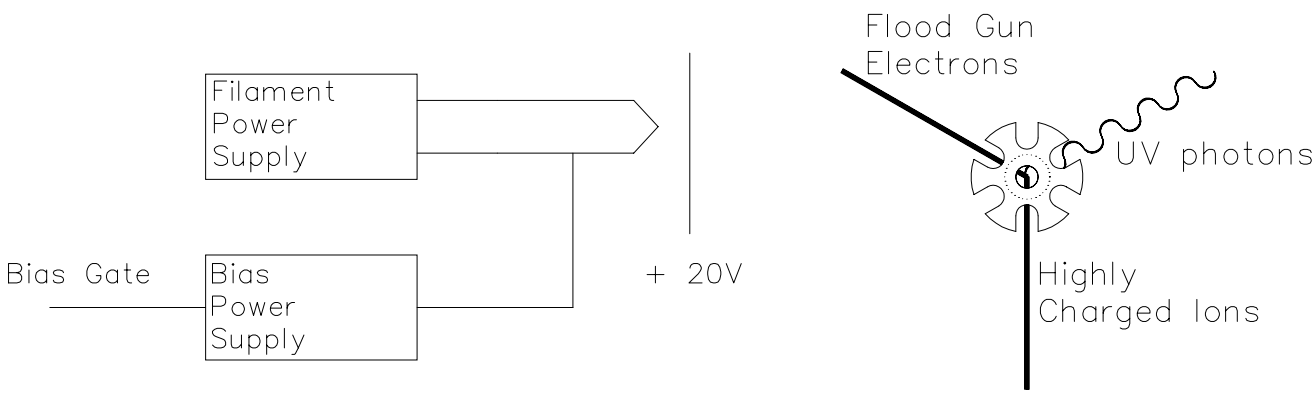

Figure 1 Layout of Sample Access (View from top)

The secondary ion/electron imaging leneseing system was designed with six radial ports providing line of sight access to the target surface. The secondary ions/electrons are collected from the center of the sample and imaged (out of the page) through the magnification optics onto the position sensitive detector. At present three of the access ports are used; one for the primary ion excitation, one for the UV excitation, and one for the electron flood gun neutralization. The physical layout of the target interaction region is shown from the view point of the position sensitive detector in Figure 1 (right).

\section{RESLUTS}

To demonstrate the operation of the electron flood gun we have imaged an $\mathrm{L}$ shaped scratch on a glass microscope slide. This microscope slide was cleaned with achol and the scratch was made with a diamond scribe. The image is shown on the left of figure 2 is of the apex of the $\mathrm{L}$ at a magnification of $40 \mathrm{x}$ (field of view is $950 \mu \mathrm{m}$ ) and the image on the right is near the apex of the $\mathrm{L}$ at a magnification of $\approx 90 \mathrm{x}$ (field of view is $400 \mu \mathrm{m}$ ). The images obtained with out the flood gun compensation on showed no contrast from the scratch. Secondary ion time-of-flight spectra were collected from both the undamaged and damaged areas of the sample. The undamaged areas of the sample showed higher rations of $\mathrm{C}_{2} \mathrm{H}_{\mathrm{x}=0-2}$ to $\mathrm{SiO}_{\mathrm{x}}$ as would be expected from areas where the surface hydrocarbons have not been removed. Time-offlight spectra showing negative ions from the undamaged and damaged areas of the sample are shown in figure 3. To demonstrate an interesting feature of this analysis process we point out that there is no boron present in the T-O-F spectra indicating that the microscope slide we employed in this test was not Pyrex/BoroSilicate glass. 

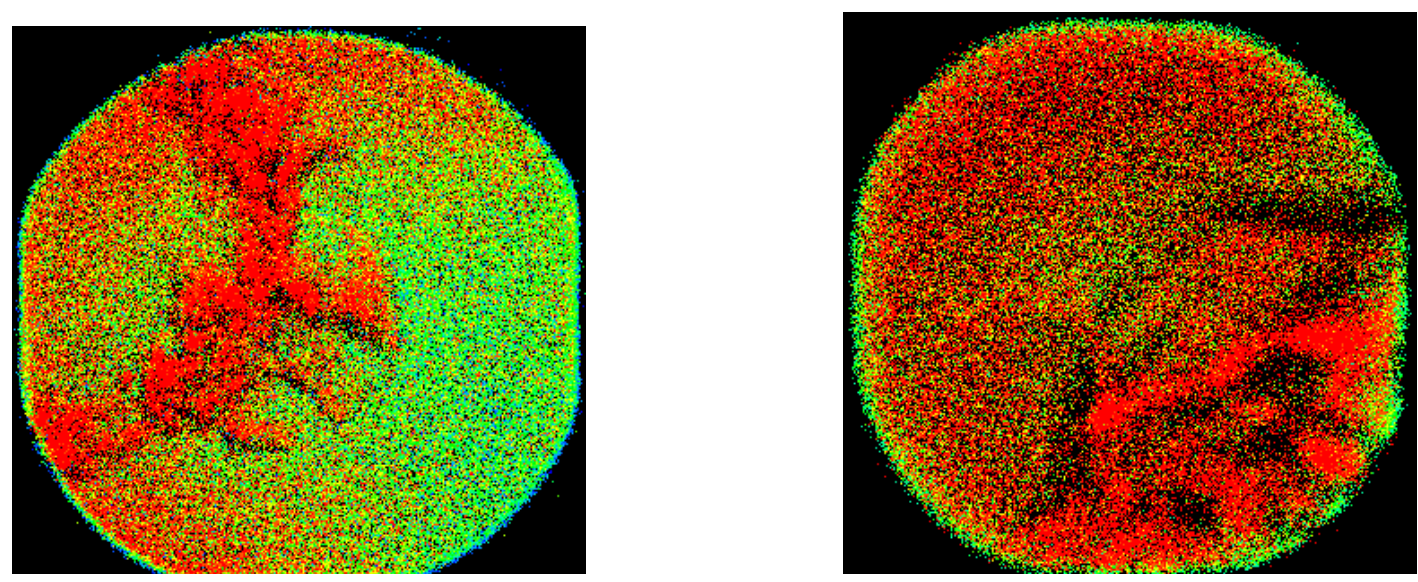

Figure 2 Left side Apex of $L$ scratch on microscope slide at 40x, right side near Apex of scratch at magnification of 90x.

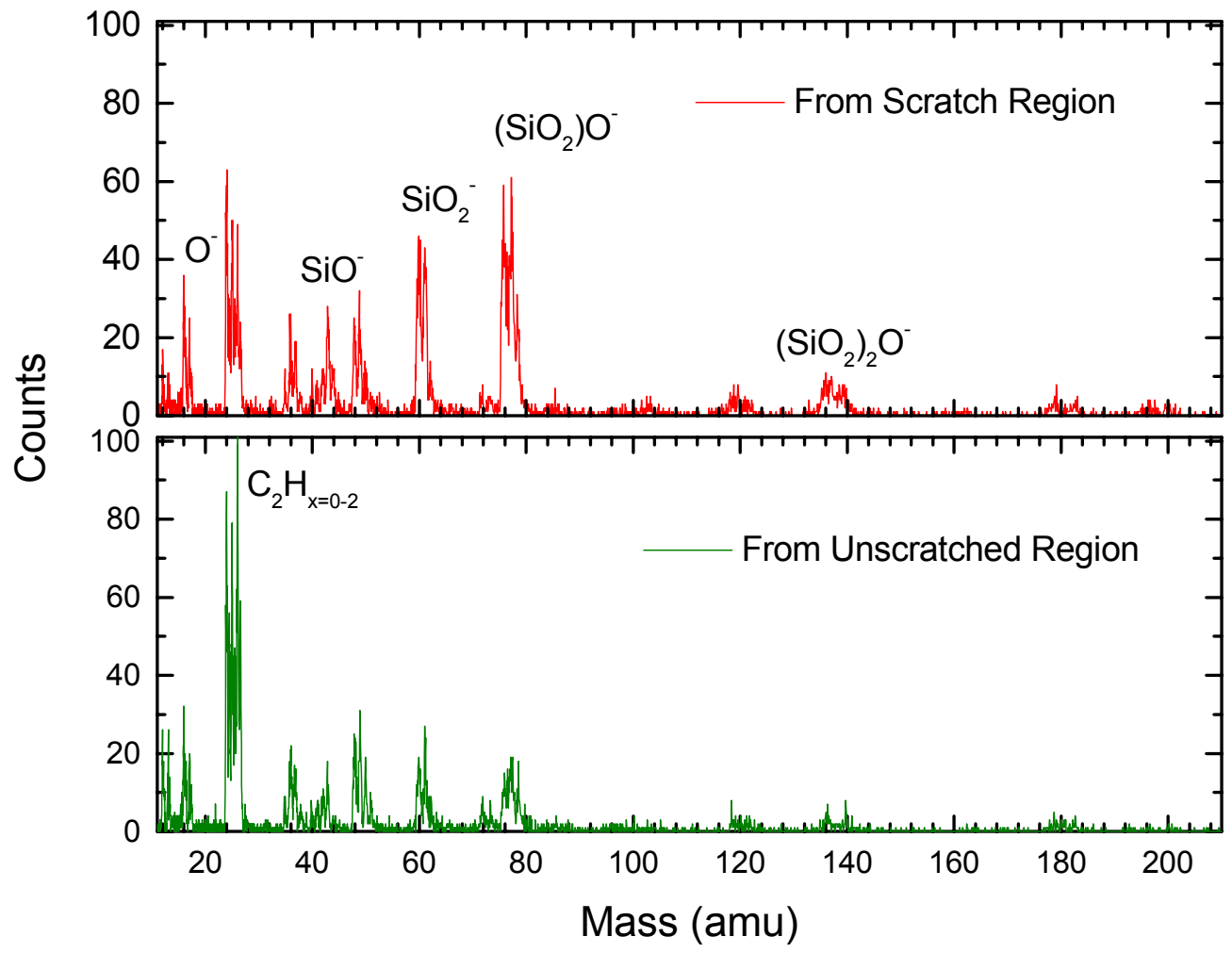

Figure 3 Glass Slide time-of-flight spectra, top from scratched region, bottom from unscratched region. Note the higher ratios of $\mathrm{C}_{2} \mathrm{H}_{\mathrm{x}=0-2}$ to $\mathrm{SiO}_{\mathrm{x}}$ from the unscratched region.

As a second demonstration of the effectiveness of the electron flood gun we imaged the surface of a pressed powder of a high explosive (poor electrical conductor). Initial imaging tests on this bare surface proved unusable due to surface charging. A first attempt to improve the image and T-O-F SIMS spectra was to press an $18 \mu \mathrm{m}$ (spacing) nickel grid into the surface to dissipate the charge buildup. The image on the left side of figure 4 shows an image of the high explosive with the grid pressed into the surface. The metal grid can clearly be seen as areas of high electron emission (red), an area of positive charge at the 
center of the grid causes and apparent area of high electron emission. This apparent area of high emission is due to a focusing effect of the positively charged surface. The right side of figure 4 shows the same target with the electron flood gun turned on. The charged induced focusing effect is has been eliminated greatly improving the image resolution. T-O-F spectra from the same surface is shown in figure 5 where the top is without charge compensation and the bottom is with charge compensation. The improvement in the resolution of the T-O-F spectrum is apparent. The water peak is well defined and the $\mathrm{C}_{2}, \mathrm{C}_{2} \mathrm{H}$, and $\mathrm{C}_{2} \mathrm{H}_{2}$ series of peaks are clearly distinct.
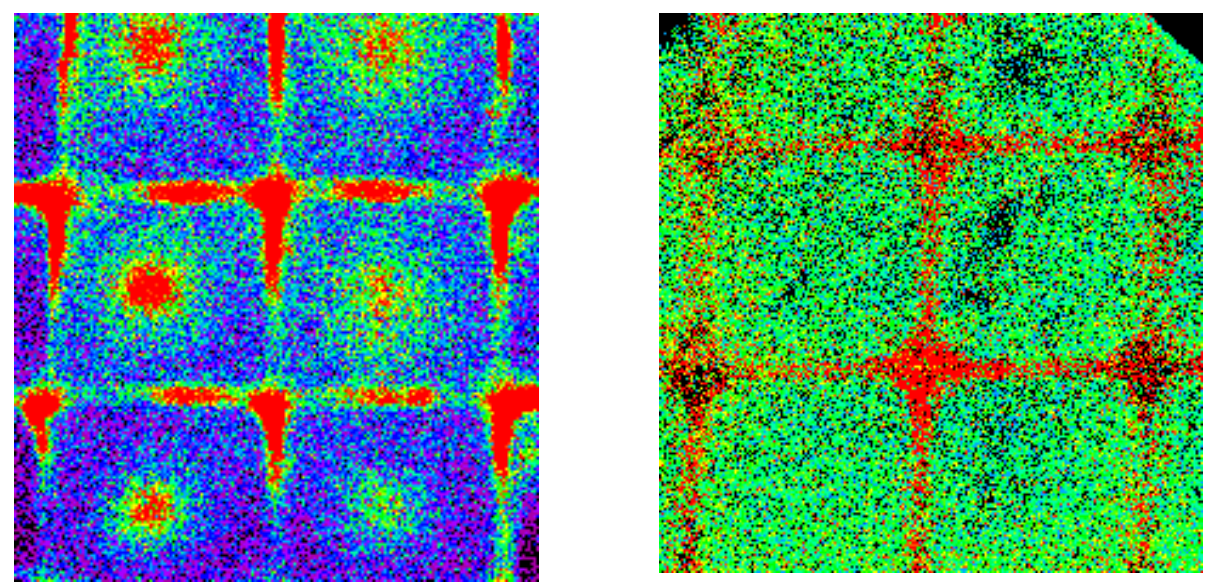

Figure 4 High explosive matrix with a $18 \mu \mathrm{m}$ nickel grid pressed onto the front surface. Left side is without, right side with charge compensation.

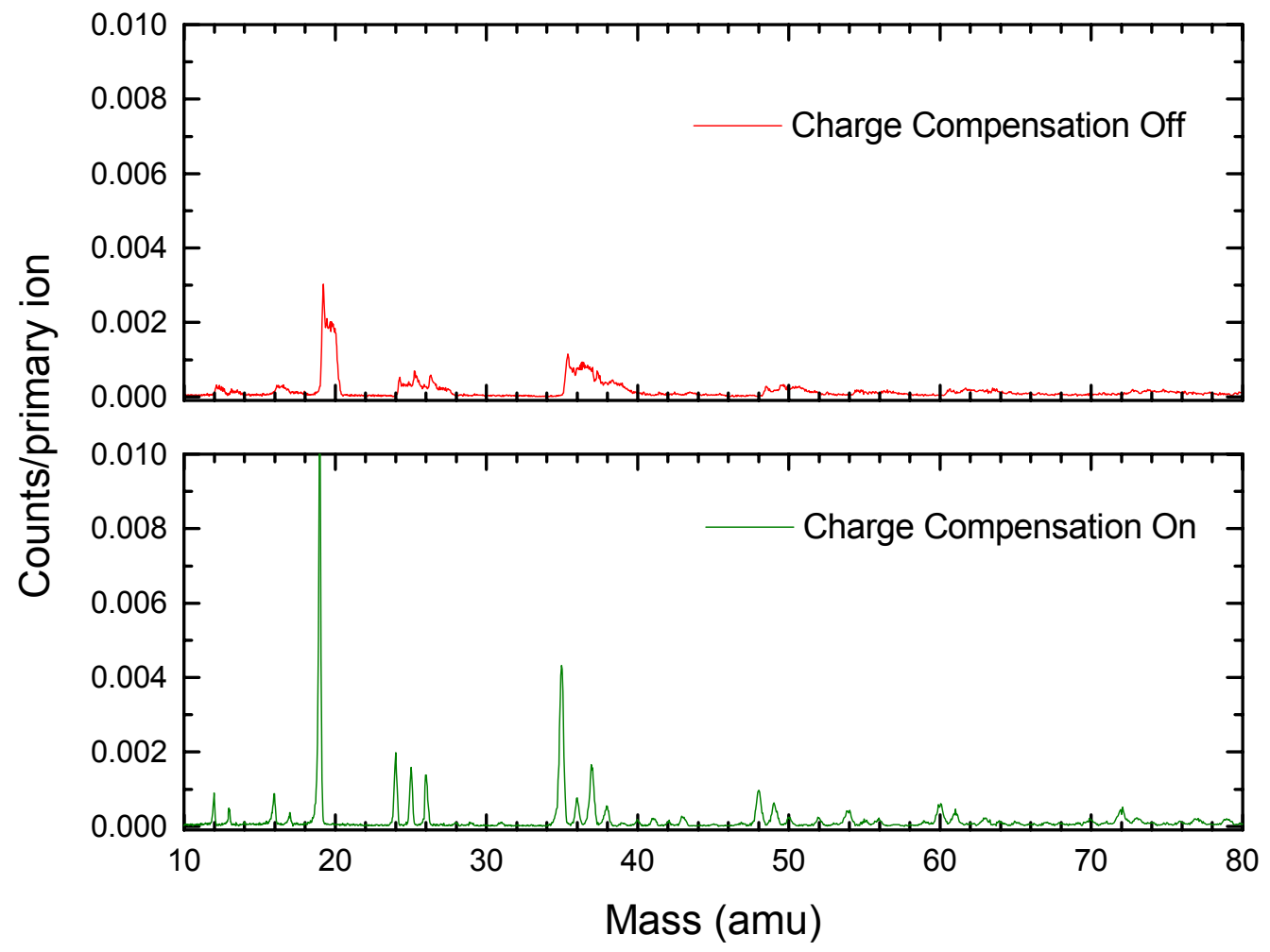


Figure 5 Charge compensation effects on T-O-F Spectrum of High explosive (insulating matrix) with $18 \mu \mathrm{m}$ metallic grid pressed into surface

By biasing the sample positively we were able to collect and image positive secondary ions from the sample. A positive ion image from the end of scratch on the microscope slide, with a magnification of 40x (field of view is $950 \mu \mathrm{m}$ ) is shown in figure 6. As with the negative ion image the positive images with out charge compensation show no contrast. Figure 7 shows a positive ion T-O-F- spectra from the same microscope slide. The top spectrum is from the unscratched region, and the bottom spectrum is from the scratched region. Note the increase in sodium and the decrease in aluminum from the scratched region.

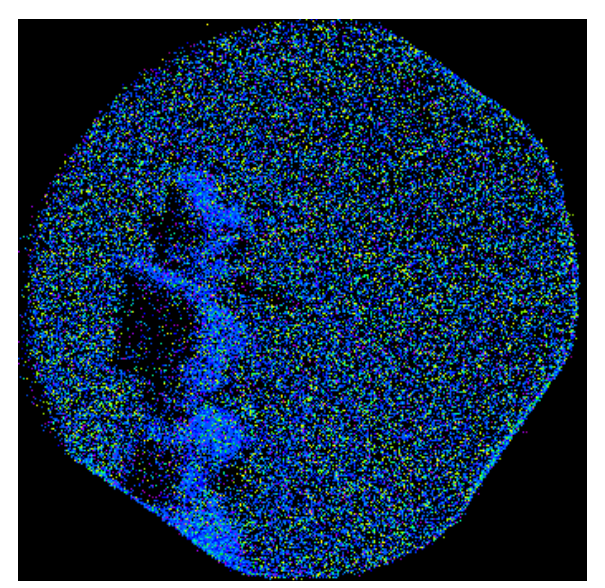

Figure 6 Positive Ion Image of the end of the $L$ scratch at $40 X$, field of view is $950 \mu \mathrm{m}$ 


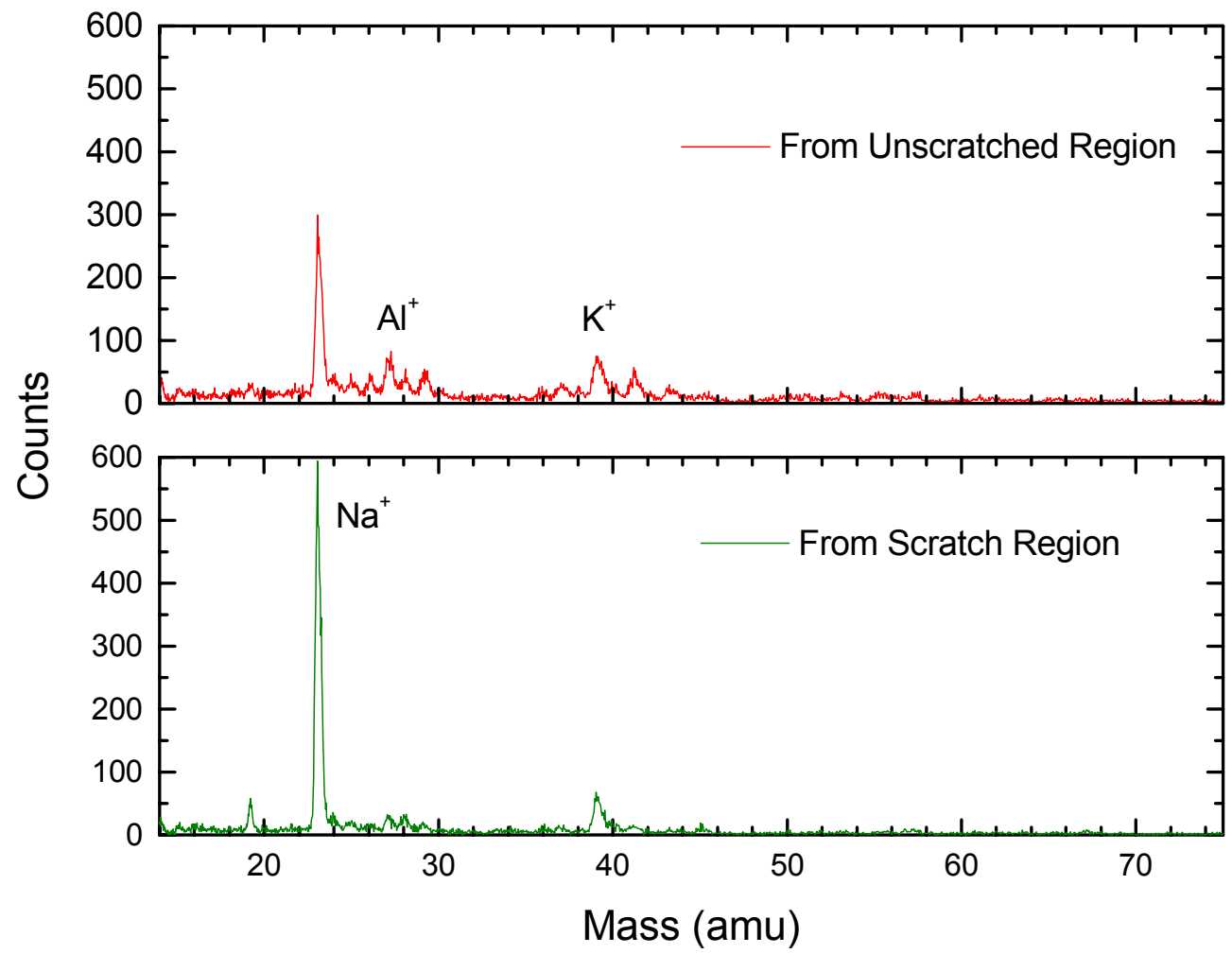

Figure 7 Positive ion T-O-F- spectra from microscope slide. Top unscratched region, bottom scratched region. Note the increase in sodium and the decrease in aluminum from the scratched region.

While the spatial resolution of this unique device could be improved, the addition of electron flood gun charge compensation, has greatly extended the utility of this HCI T-O-F microscope by allowing the analysis and imaging of insulators and semiconductors.

This work was performed under the auspices of the U.S. Department of Energy by University of California Lawrence Livermore National Laboratory under Contract No. W-7405-ENG-48.

\footnotetext{
${ }^{1}$ Alex V. Hamza, Alan V. Barnes, Ed Magee, Mike Newman, Thomas Schenkel, Joseph W. McDonald, and Dieter H. Schneider, Rev. Sci. Instr. 71, 2077 (2000).

${ }^{2}$ O. H. Griffith and W. Engel, Ultramicroscopy 36, 1 (1991).

${ }^{3}$ H, Düker, Z. Metallkd. 51, 314 (1960).

${ }^{4}$ R. Castiang and G. Slodzian, Proceedings of the European Regional Conference on Electron Microscopy (Nederlanse Vereniging voor Electron Microscopic, Delft, 1960), Vol. 1. P. 169.

${ }^{5}$ T. Schenkel, M. A. Briere, H. Schmidt-Böcking, K. Bethge, and D. H. Schneider, Phys. Rev. Lett. 78, 2481 (1997).

${ }^{6}$ J. McDonald, D. Schneider, M. Clark, and D. DeWitt, Phys. Rev. Lett. 46, 2297 (1992).

${ }^{7}$ F. Aumayr, H.Kurz, D. Schneider, M. Briere, J. W. McDonald, C. E. Cunningham, and H. P. Winter, Phys. Rev. Lett. 71, 1943 (1993).

${ }^{8}$ T. Schenkel, A. V. Barnes, M. A. Briere, A. Hamza, A. Schach von Wittenau, and D. Schneider, Nucl. Instrum. Methods Phys. Res. B 125, 153 (1997).

${ }^{9}$ T. Schenkel, A. V. Barnes, A. V. Hamza, D. H. Schneider, J. C. Banks, and B. L. Doyle, Phys. Rev. Lett. 80, 4325 (1998).

${ }^{10}$ G. F. Rempfer and O. H. Griffith, Ultramicroscoppy 47, 35 (1992).
} 
${ }^{11}$ D. Schneider, M. W. Clark, B. M. Penetrante, J. McDonald, D. DeWitt, and N. J. Bardsley, Phys. Rev. A 44, 3119 (1991). 